\title{
Mathematical Model for Mump Disease in Thailand
}

\author{
P.Pongsumpun
}

\begin{abstract}
The infection caused by mumps virus, called as Mumps. This disease is transmitted by respiratory system from an infected person. Mumps virus passes from one person to another by saliva and nasal secretions. This disease can be occurred both children and adult. In this paper, we formulate the mathematical for mumps disease in Thailand. The analytical solutions of the model are given. The numerical solutions are shown to explain the behavior of the disease.
\end{abstract}

Keywords-Mathematical model, mumps virus, numerical analysis.

\section{INTRODUCTION}

Mumps is a disease caused by a viral infection that occurs in school-age children. Most patients often have swelling and pain in the salivary glands, especially the salivary glands.This disease is mild but sometimes causes serious illness. Although the mumps vaccine can reduce the mumps infection, but there are the report of mumps patients in many countries such as United Kingdom, Canada and United States. Mumps virus caused by a paramyxovirus, a member of the Rubulavirus family. The incubation period for Mumps is about 12 to 25 days[1]. After infection, some illness has no symptoms or very mild symptoms but they can still transfer the virus to the others.Mumps is a worldwide disease. In the United States, before the introduction of mumps vaccines in 1967. There were the mumps outbreaks every $2-5$ years. It can be found throughout the year. The peak of incidence occurs during January-May. The outbreak is often occurs in the place with dense communities such as schools, colleges, camps, boarding houses, etc. After 1967, the incidence of mumps has decreased by more than 99 percent from Report of the Department of Disease Control United States.From 2001 to 2005, there is the mumps patients about 265 persons per year. There is the report of outbreak for many countries such as Netherlands, United Kingdom, United states, Canada. There were the mumps outbreaks in 2006 about 6,584 persons. The highest incidence occurs during 18-24 years. There are $83 \%$ of mumps cases are found in universities. The strain of mumps virus found in patients is genotype G16. There is only $13 \%$ of mumps who did not get vaccination. About $63 \%$, the mumps patients got 2 doses of mumps vaccine. The vaccines inefficient during the outbreak due to many factors, such as the decline in the level of immunity,

Manuscript received April 5, 2019.

P.Pongsumpun is with Department of Mathematics, Faculty of Science, King Mongkut's Institute of Technology Ladkrabang,Chalongkrung road, Ladkrabang, Bangkok 10520. the coexistence between patients and those at high risk of infection, such as slums, hostels, etc. Mumps vaccine is also the most important way to control the outbreak of mumps. The epidemic of outbreak were rapidly decrease in 2008. The report of patients were found only 376 persons. During 2009 - 2010, there were 1,521 mumps cases. The first suspected patient were 11-year-old Jewish boy traveling to a summer camp[2].

In Thailand [3], mumps have been monitored since 1971. The incidence has increased since the beginning of 1997 until the combined vaccine against measles, mumps, rubella (MMR) was initiated to Thai children. Nationwide mcausing the incidence of mumps periodic outbreaks have been found in those who have not been vaccinated. Mumps are less common in young children under 1 year because they are immune to mumps virus. They received the virus from the mother through the placenta. Before the use of vaccines against mumps, more than 50 percent of the disease occurs in children aged 5 - 9 years and 90 percent of patients younger than 14 years old. It was found that $80-90$ percent of adults over the age of 20 are immune to mumps from natural infections. After the mumps vaccine in 2001, there were $49 \%$ of patients older than 15 years old immune to mumps virus that is caused by vaccination. Females and males have the different rates of mumps infection. Mumps virus is found only in people. Mumps is a common disease in winter and early summer. It usually occurs in early childhood, ages $2-5$ years, but it is mostly found in adolescence. It can be found in both females and males.Mumps is caused by a virus in Paramyxoviris group.The disease can be contacted between each other directly by breathing and through the patient's saliva such as water and food by using a container together. The disease occurs in children with all ages. If the disease occurs in adults, there will be severe symptoms.Qu et al.[4] formulated the model for mumps disease with season in China.Hammami et al.[5] used mathematical model to study the mumps with vaccination in Scotland. Li et al. [6] constructed and analyzed long-term mumps surveillance data of an SVEILR (susceptible-vaccinated-exposed-severely

infectious-mildly infectious-recovered) model with optimized parameter values to describe the mumps infections in China. In this study, we formulate and analyze the transmission of Mumps in Thailand by standard dynamical modeling method. The equilibrium states and stability conditions for each state are determined. Numerical analysis of our model are shown to confirm our analytical results. The behavior of each population is shown for the different parameters to be the way for reducing the outbreak of this virus. 


\section{MATHEMATiCAL Model}

Our model considers the transmission of Mumps virus between human by separating the human population into 4 classes such as susceptible(S), Exposed (E), Infectious(I) and Recovered classes(R).In the beginning, the human has no virus. After he or she is infected with mumps virus from the mumps cases, he or she can be exposed. After the incubation time is passed, the mumps cases can transmit the virus to the susceptible human. We use SEIR model for describing the transmission of this disease

$$
\begin{aligned}
\frac{d s}{d t} & =\mu N-D S-\beta S I \\
\frac{d E}{d t} & =\beta S I-D E-\gamma E \\
\frac{d I}{d t} & =\gamma E-D I-v I \\
\frac{d R}{d t} & =v I-D R
\end{aligned}
$$

with the condition $N=S+E+I+R$

The parameters in our model are define in Table 1 .

TABLE I. THE DEFINITIONS OF VARIABLES/PARAMETERS OF EQUATIONS(1) TO

Variables/parameters

(4).

$\begin{array}{ll}S & \begin{array}{l}\text { Number of susceptible mumps } \\ \text { infection }\end{array} \\ E & \text { Number of exposed mumps infection } \\ I & \text { Number of mumps infectious cases } \\ R & \text { Number of recovered human } \\ N & \text { Total human population } \\ \mu & \text { Birth rate of human population } \\ d & \text { Death rate of human population } \\ \beta & \text { Infection rate of mumps virus } \\ \gamma & \text { Incubation rate of Mumps virus } \\ v & \text { Recovery rate }\end{array}$

\section{A. Analytical results}

The standard dynamical analysis is used in this model. The equilibrium states are found by setting the time rate of change for each population to be zero, i.e.

$d S / d t=0, d E / d t=0, d I / d t=0, d R / d t=0$

Then the equilibrium states are

i) The disease free state:

$$
\left(\mathrm{S}^{*}, E^{*}, \mathrm{I}^{*}, \mathrm{R}^{*}\right)=\left(\frac{\mu N}{D-C_{S}}, 0,0,0\right)
$$

ii) The endemic state $\left(S^{*}, E^{*}, I^{*}, R^{*}\right)$ :

where

$S^{*}=\frac{(D+\gamma)(D+v)}{\beta \gamma}$

$$
\begin{aligned}
& E^{*}=\left(-\frac{D^{3}-\beta \mu N \gamma+D \gamma v-C_{S}(D+\gamma)(D+v)+D^{2}(\gamma+v)}{\beta \gamma(D+\gamma)}\right) \\
& I^{*}=\left(-\frac{D^{3}-\beta \mu N \gamma+D \gamma v-C_{S}(D+\gamma)(D+v)+D^{2}(\gamma+v)}{\beta(D+\gamma)(D+v)}\right) \\
& R^{*}=-v\left(\frac{D^{3}-\beta \mu N \gamma+D \gamma v-C_{S}(D+\gamma)(D+v)+D^{2}(\gamma+v)}{\beta D(D+\gamma)(D+v)}\right) .
\end{aligned}
$$

The stability of each equilibrium state is determined by the sign of eigenvalues. If the eigenvalues have negative real parts, then that equilibrium states wil be stable. The eigenvalues are found by solving the characteristic equation:

$$
\operatorname{det}\left(\lambda I_{4}-J_{f}\right)=0
$$

where $\mathrm{J}_{\mathrm{F}}$ is the Jacobian matrix and $\mathrm{I}_{4}$ is the identity matrix dimension $4 \mathrm{x} 4$.

$$
I_{4}=\left[\begin{array}{llll}
1 & 0 & 0 & 0 \\
0 & 1 & 0 & 0 \\
0 & 0 & 1 & 0 \\
0 & 0 & 0 & 1
\end{array}\right]
$$

The Jacobian of our equations is given by

$$
\mathrm{J}_{\mathrm{F}}=\left[\begin{array}{cccc}
-(D+\beta I) & 0 & -\beta S & 0 \\
\beta I & -D-\gamma & \beta S & 0 \\
0 & \gamma & -D-v & 0 \\
0 & 0 & v & -D
\end{array}\right] .
$$

We have

$$
\lambda I_{4}-J_{f}=\left[\begin{array}{cccc}
\lambda-(-(D+\beta I)) & 0 & \beta S & 0 \\
-\beta I & \lambda-(-D-\gamma) & -\beta S & 0 \\
0 & -\gamma & \lambda-(-D-v) & 0 \\
0 & 0 & -v & \lambda-(-D)
\end{array}\right] \text {. }
$$

The characteristic equation is

$$
\begin{aligned}
\operatorname{det}\left(\lambda I_{4}-J_{f}\right) & =(\lambda+D)\left(C_{44}\right) \\
& =(\lambda+D)(-1)^{4+4} M_{44} \\
& =(\lambda+D) M_{44} \\
& =(\lambda+D)\left|\begin{array}{ccc}
\lambda+D+\beta I & 0 & \beta S \\
-\beta I & \lambda+D+\gamma & -\beta S \\
0 & -\gamma & \lambda+D+v
\end{array}\right| \\
& =(\lambda+D)\left[\begin{array}{l}
(\lambda+D+\beta I)(\lambda+D+\gamma)(\lambda+D+v)+(\beta S)(-\beta I)(-\gamma) \\
-(-\gamma)(-\beta S)(\lambda+D+\beta I)
\end{array}\right] \\
& =0
\end{aligned}
$$

From (5), we have

$$
\begin{aligned}
& \lambda+D=0 \text { or } \lambda_{1}=-D \\
& \text { and }\left[\begin{array}{l}
(\lambda+D+\beta I)(\lambda+D+\gamma)(\lambda+D+v)+(\beta S)(-\beta I)(-\gamma) \\
-(-\gamma)(-\beta S)(\lambda+D+\beta I)
\end{array}\right]=0 \\
& \text { or }
\end{aligned}
$$




$$
\left[\begin{array}{l}
\lambda^{3}+3 D \lambda^{2}+\beta I \lambda^{2}+\gamma \lambda^{2}+v \lambda^{2}+2 \beta D I \lambda+\beta I \gamma \lambda \\
+2 D \gamma \lambda+2 D v \lambda+3 D^{2} \lambda+\beta I v \lambda+\gamma v \lambda-\beta \gamma S \lambda \\
+D^{3}+\beta D^{2} I+D^{2} \gamma+D^{2} v+\beta I D \gamma+\beta I D v+D \gamma v \\
-\beta \gamma S D+\beta I v \gamma
\end{array}\right]=0
$$

Case 1: Disease free state:

The remaining eigenvalues are found by solving the equation $\left[\begin{array}{l}\lambda^{3}+3 D \lambda^{2}+3 D^{2} \lambda+\gamma \lambda^{2}+2 D \gamma \lambda+D^{3}+D^{2} \gamma+v \lambda^{2} \\ +2 D \lambda \nu+D^{2} v+\gamma v \lambda+D \gamma v-\frac{\beta \gamma \lambda \mu N}{D}-\beta \gamma \mu N\end{array}\right]=0$.

Therefore $\lambda_{2}=-D$ and

$\lambda_{3}=\frac{-2 D-\gamma-v-\sqrt{\gamma^{2}+\frac{4 \beta \mu N \gamma}{D}-2 \gamma v+v^{2}}}{2}$

is negative when $\gamma^{2}+\frac{4 \beta \mu N \gamma}{D}-2 \gamma \nu+v^{2}>0$

$$
\lambda_{4}=\frac{-2 D-v-\gamma+\sqrt{\gamma^{2}+\frac{4 \beta \mu N \gamma}{D}-2 \gamma \nu+v^{2}}}{2}
$$

is negative when $\gamma^{2}+\frac{4 \beta \mu N \gamma}{D}-2 \gamma \nu+v^{2}>0$ and

$$
\frac{2 D+v+\gamma}{2}>\frac{1}{2} \sqrt{\gamma^{2}+\frac{4 \beta \mu N \gamma}{D}-2 \gamma v+v^{2}} .
$$

We can see that the disease free state is stable when

$$
\begin{gathered}
\gamma^{2}+\frac{4 \beta \mu N \gamma}{D}-2 \gamma \nu+v^{2}>0 \text { and } \\
\frac{2 D+v+\gamma}{2}>\frac{1}{2} \sqrt{\gamma^{2}+\frac{4 \beta \mu N \gamma}{D}-2 \gamma \nu+v^{2}} .
\end{gathered}
$$

Case2: The endemic state:

After we substitute the endemic state,from(5) we have $\lambda+D=0$ or $\lambda_{1}=-D$,

The remaining eigenvalues are found from solving (6), they have negative eigenvalues when the following conditions are satisfied.

$$
\begin{gathered}
\lambda_{2}<0 \text { when } A-B<2 C(D-C) \\
\lambda_{3}=\frac{-(A+B)}{2 C}-C-D-\frac{\sqrt{3}(A+B)}{2 C}-C-D \\
\lambda_{3}<0 \text { when }-(A+B)-\sqrt{3}(A+B)<4 C(D+C) \\
\lambda_{4}=\frac{-(A+B)}{2 C}-C-D+\frac{\sqrt{3}(A+B)}{2 C}-C-D \\
\lambda_{4}<0 \text { when }-(A+B)+\sqrt{3}(A+B)<4 C(D+C)
\end{gathered}
$$

\section{B. Numerical simulations.}

The numerical simulations[7-9] are present in this section by using the parameters satisfied to each condition as follows:

\section{For the disease free state.}

$\mu=1 /\left(365^{*} 74\right), \mathrm{D}=1 /\left(365^{*} 74\right), \beta=1 / 8, \gamma=1 / 17$.
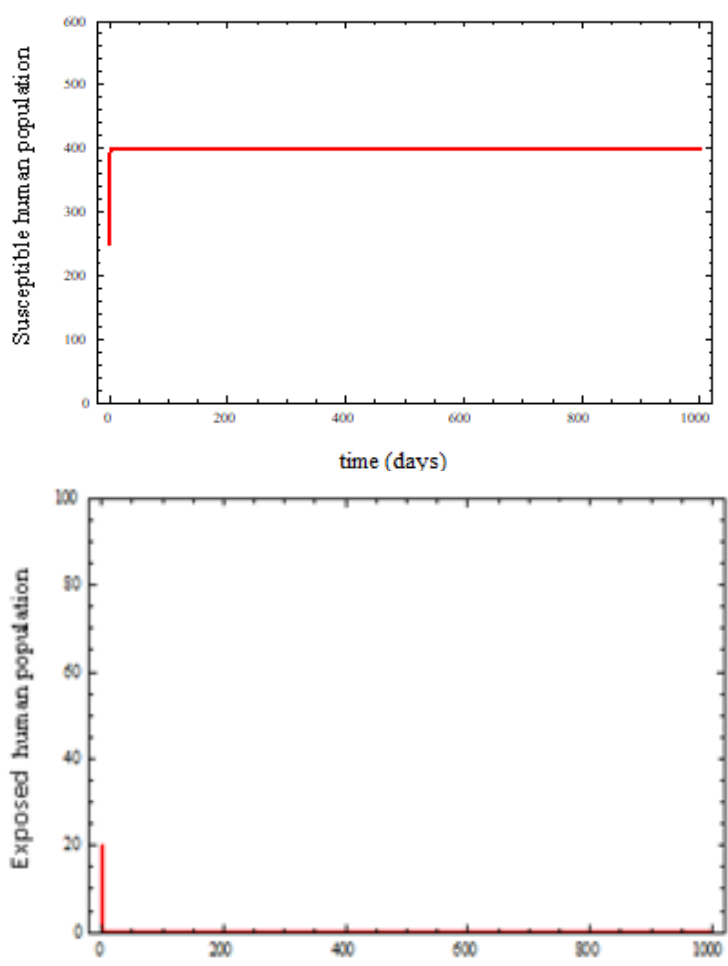

time(days)

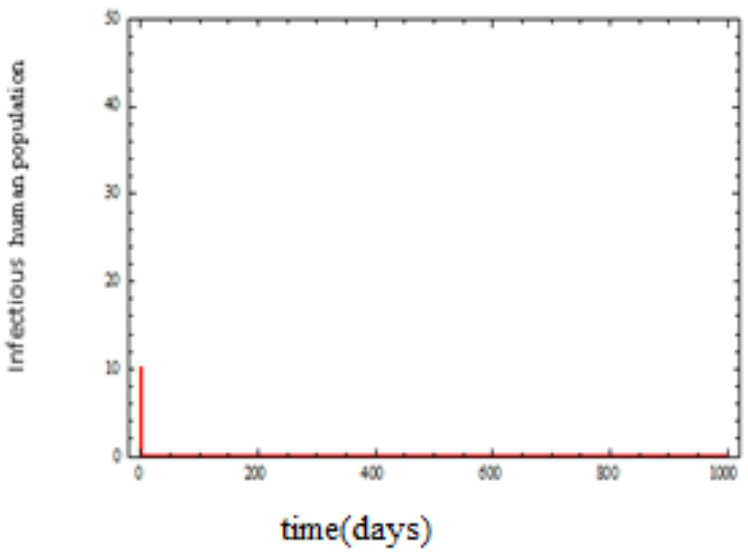




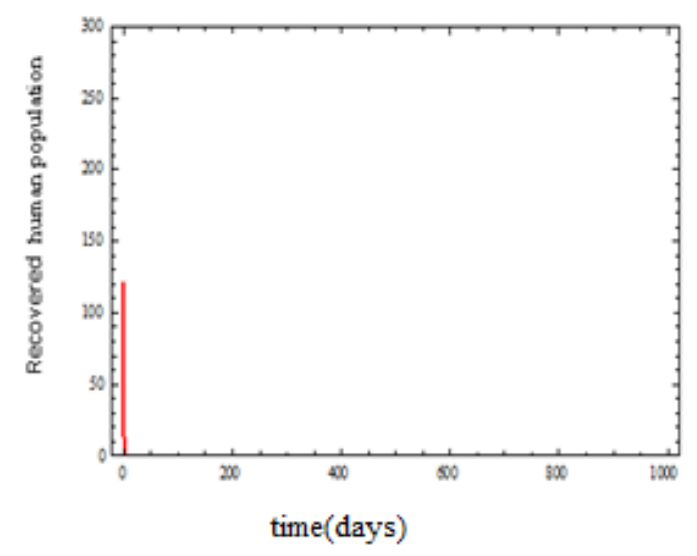

Fig.1 Numerical simulations of each human population for the disease free state. We will see that the solutions converge to the disease free state when it satisfy(7).

\section{For the endemic disease state.}

$\mu=\frac{1}{365 \times 74.18}, D=\frac{1}{365 \times 74.18}, \beta=\frac{1}{8}, \gamma=\frac{1}{17}, v=\frac{1}{14}$

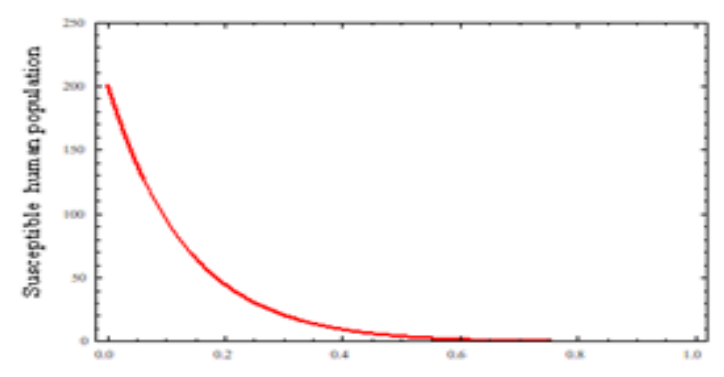

time(days)

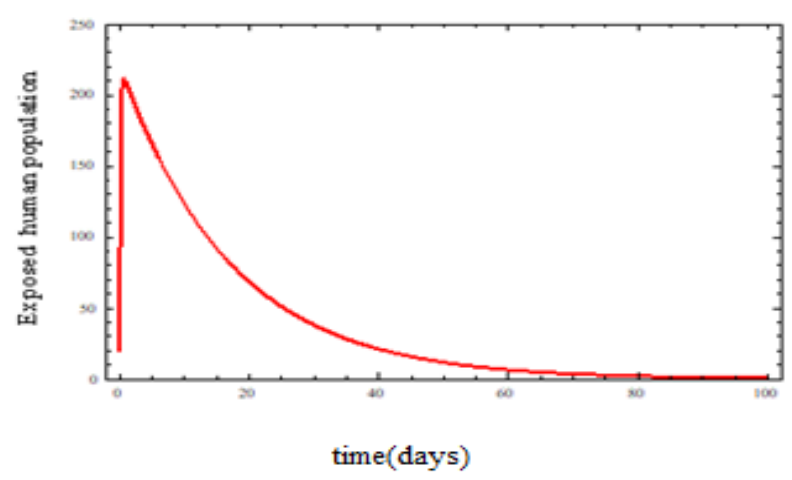

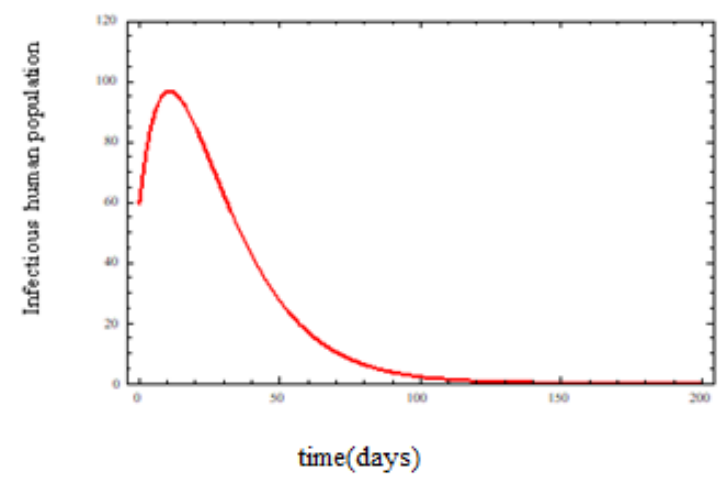

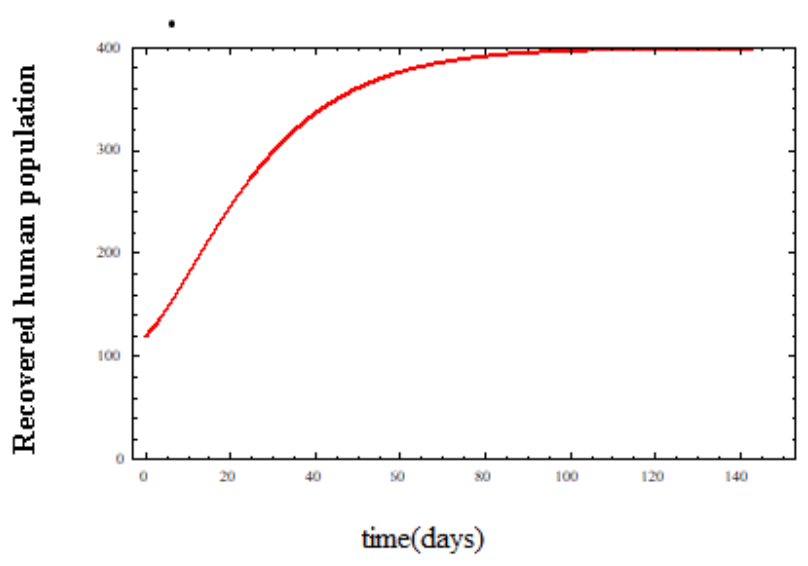

Fig.2 Numerical simulations of each human population for the disease free state. We will see that the solutions converge to the endemic disease state when it satisfies (8)-(10).

\section{Discussion AND CONCLUSIONS}

In this paper, we formulate and analyze the model for the transmission of Mumps disease. The equilibrium states of this model are defined as the disease free state and endemic state. The stability condition for the disease free state and endemic state are shown in eqs.(7)-(10).Furthermore, we simulate the behavior solution of each population for the different parameters as shown in fig.3-4.

i) For the different incubation period of mumps virus. The time series solutions of E, I and R are shown in fig.3. 

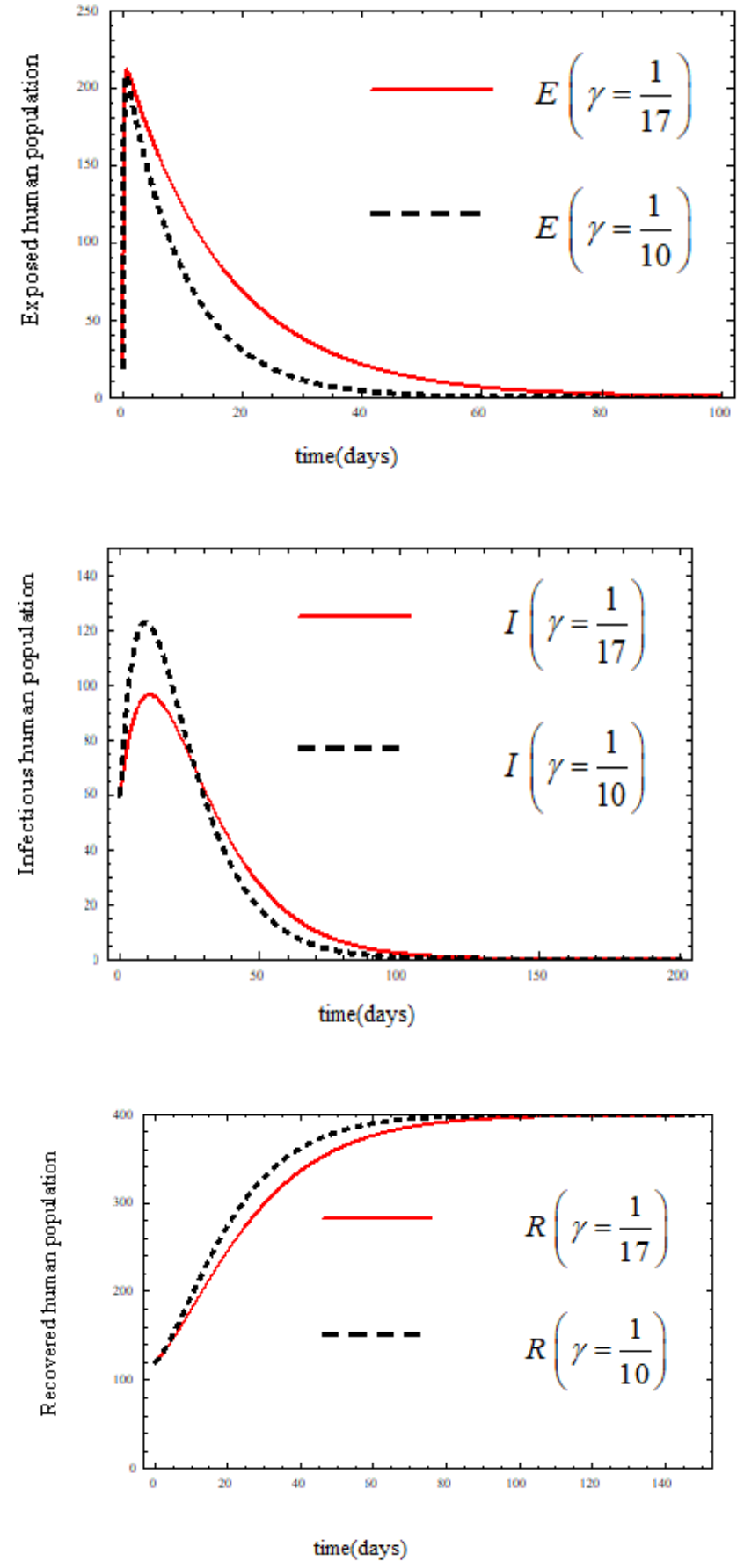

Fig.3 Numerical Simulations of exposed, infectious and recovered human population for the different period of incubation. We can see that the outburst time of epidemic is longer when the longer time of incubation.

ii) For the different period of recovery. The time series solutions of I and R are shown in fig.4.
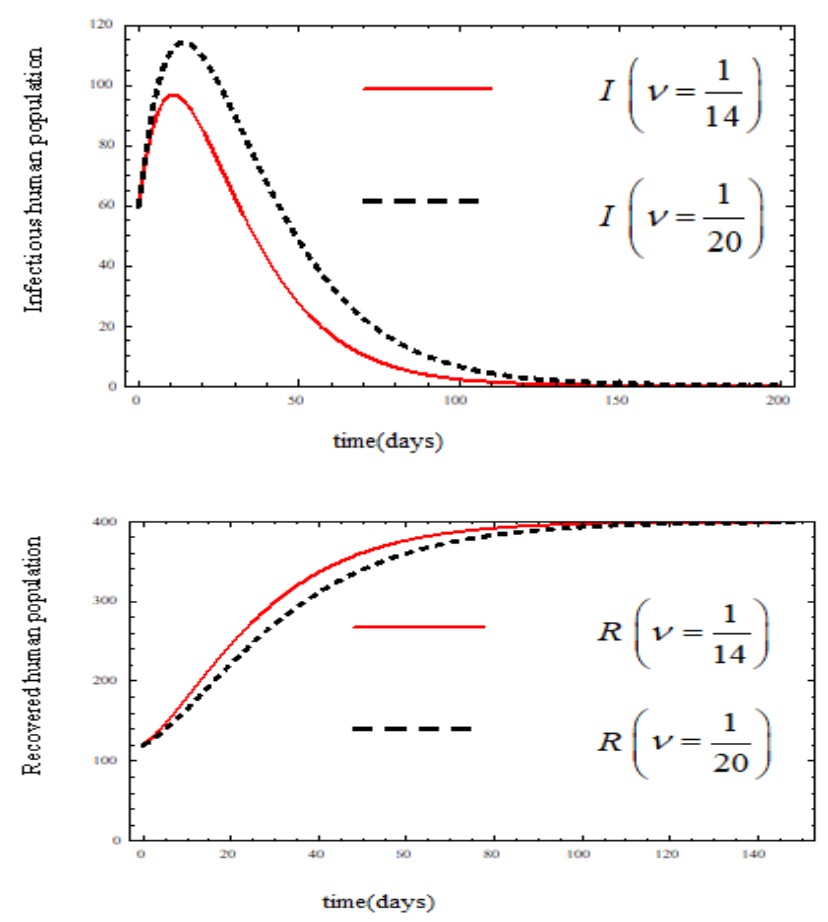

Fig.4 Numerical Simulations of exposed, infectious and recovered human population for the different period of recovery. We can see that the time of infection and recovery are longer when there is the longer time of recovery.

Mathematical models are applied to describe the transmission of many diseases[10-12]. The parameter conditions are obtained to be the way for reducing the disease outbreaks. Mumps infections are found in all ages of human in Thailand. However, the mumps vaccination should be considered in the future research.

\section{REFERENCES}

[1] https://www.cdc.gov/mumps/hcp.html

[2] Green book chapter 23, Available from https://assets.publishing.service.gov.uk/government/uploads/syste m/uploads/attachment_data/file/147975/Green-Book-Chapter-23-v 2_0.pdf

[3] http://www.boe.moph.go.th/boedb/surdata/

[4] Q. Qu,, C.Fang, L. Zhang, W. Jia, J.Weng, Y. Li, A Mumps Model with Seasonality in China, Infectious Disease modelling,/10.1016j.idm.2016.10.001

[5] D.Hamami, Ross Cameron, K.G. Pollock and C.Shankland, Waning Immunity Is Associated with Periodic Large Outbreaks of Mumps:A Mathematical Modeling Study of Scottish Data,Frontiers in Physiology, vol.8, 2017, doi: 10.3389/fphys.2017.00233]

[6] L. Yong, X.Liu and L.Wang, Modelling the Transmission Dynamics and Control of Mumps in Mainland China, Int. J. Environ. Res. Public Health 2018, 15, 33; doi:10.3390/ijerph15010033

[7] L.Esteva and C.Vargas,"Analysis of a dengue disease transmission model”, MathematicalBiosciences, vol.150, pp.132-151, 1998. https://doi.org/10.1016/S0025-5564(98)10003-2

[8] J.D.Hoffman,Numerical Methods for Engineers and Scientists, Sigapore: McGraw-Hill.

[9] L.Edelstein, Mathematical models in biology. $1^{\text {st }}$ ed. New York :Random House, 1989.

[10] J.Lamwong, I.M.Tang and P.Pongsumpun, MERS model of Thai andSouth Koreaa population, Current Applied Science and Technology Journal, 2018, 18, pp.45-57. 
[11] P.Pongsumpun, Network Distribution Model of Influenza Virus in the Community, International Journal of Modeling and Optimization, Vol. 8, No. 1, February 2018, pp.30-32. https://doi.org/10.7763/IJMO.2018.V8.620

[12] P.Chanprasopchai, I.M.Tang and P.Pongsumpun,SIR Model for Dengue Disease with Effect of Dengue Vaccination, Computational and Mathematical Methods in Medicine, Vol. 2018, Article ID 9861572, 14 pages, https://doi.org/10.1155/2018/9861572 